\title{
EL USO DE MICROVÍDEOS COMO REFUERZO DEL APRENDIZAJE PRESENCIAL EN LA ENSEÑANZA DE LAS BASES DEL CONTROL AUTOMÁTICO
}

\author{
Vicente Arevalo, Jesús María Vicente del Rey, Isabel García Morales \\ Dpto. de Ingeniería de Sistemas y Automática, Universidad de Málaga, Andalucía Tech \\ \{varevalo,jmvicente, isabelgm\}@uma.es
}

\section{Resumen}

Este artículo describe una experiencia de innovación educativa realizada durante el curso 2017/2018 en el Dpto. de Ingeniería de Sistemas y Automática de la Universidad de Málaga. Esta experiencia ha consistido en el empleo de microvídeos como herramienta de apoyo para el aprendizaje de los conceptos básicos del control automático de sistemas en tiempo continuo por parte de los alumnos de 14 asignaturas de diferentes titulaciones.

Por lo general, los alumnos encuentran grandes dificultades en dicho aprendizaje, de ahi que un aspecto clave en esta experiencia haya sido la búsqueda de estrategias para que los materiales elaborados resultaran amenos y atractivos, a la vez que didácticos.

Para facilitar, propiciar y analizar el uso de los mismos, se han aprovechado las ventajas que supone su publicación en una plataforma tal como YouTube. El canal www.youtube.com/automaticabasica, en el que se ha incluido el material elaborado, cuenta en la actualidad con 39 microvídeos, 9 listas de reproducción (bloques conceptuales), un total de 564 suscriptores, $\quad 55.325 \quad$ reproducciones $y$, aproximadamente, 78 días de tiempo acumulado de reproducción. Los resultados obtenidos, aunque son muy preliminares, muestran un impacto positivo en el rendimiento académico de los alumnos que los han utilizado.

Palabras clave: control automático, aprendizaje autónomo, microvídeo, YouTube, MATLAB, innovación educativa.

\section{INTRODUCCIÓN}

El aprendizaje de los conceptos básicos del control automático es fundamental en la formación de cualquier ingeniero, de ahí que la práctica totalidad de los estudios conducentes a la obtención de un título de grado en el ámbito de las ingenierías cuenten con una o más asignaturas relacionadas con este tema. Sólo en la Universidad de Málaga (UMA), el número de titulaciones de enseñanzas técnicas que incluyen en sus planes de estudios asignaturas en las que se abordan contenidos relacionados con el control automático ronda la quincena, lo que da una idea de la importancia que supone el estudio de esta materia en ese tipo de titulaciones.

El objetivo fundamental de estas asignaturas es lograr que el alumno conozca y aprenda los conceptos básicos de los sistemas de control automático. El estudio de este tipo de sistemas tiene en las mismas su punto de partida en la determinación de un modelo matemático del proceso que se pretende controlar y el análisis de sus características temporales y frecuenciales y culmina con el aprendizaje de técnicas clásicas de diseño de sistemas de control. La profundidad con la que se abordan estos conceptos varía en función de la titulación y el curso en que se ubica cada una de estas asignaturas, pero son muchos los bloques conceptuales que comparten.

Por otra parte, la importante carga teórico-práctica de estas asignaturas, la amplitud de conceptos que involucran, las estrechas relaciones existentes entre ellos que impiden el aprendizaje de unos si aún no se han adquirido muchos otros, la necesidad de utilizar herramientas informáticas que facilitan la resolución de determinados problemas, etc. son cuestiones que tradicionalmente plantean dificultades a los alumnos. Ante esta situación, han sido diversas las iniciativas puestas en marcha a lo largo del tiempo en el Dpto. de Ingeniería de Sistemas y Automática (ISA) de la UMA, responsable de la impartición de estas asignaturas, que estaban destinadas a facilitar el aprendizaje de estos contenidos, pero no han tenido mucho éxito.

En este trabajo se presenta una nueva iniciativa, enmarcada en un Proyecto de Innovación Educativa financiado por la UMA, que consiste en introducir y fomentar el uso de los microvídeos (vídeos con una duración media que ronda los $5 \mathrm{~min}$.) como herramienta para el aprendizaje de esta materia. Se ha recurrido al uso de vídeos, entre otras razones, porque el consumo de vídeo bajo demanda (VOD) ha crecido sustancialmente en los últimos años (se estima que a la plataforma YouTube se suben 100 horas de vídeo cada minuto), convirtiéndose éste, de 
facto, en un medio de comunicación globalmente aceptado y muy utilizado por el alumnado [12].

Los autores están convencidos, y así lo refrendan los resultados obtenidos, de que, en la actualidad, los alumnos quieren (esperan) aprender del mismo modo en que se comunican, se informan, se divierten,...; prefieren materiales atractivos, directos, "sin libros" y "sin profesor" [2][3]. Adaptarse a esto, aunque sea mínimamente, requiere hacer cambios en el proceso de enseñanza-aprendizaje según la forma tradicional. Por otro lado, resulta evidente que hay contenidos que no encajan en esta idea $y$, en cualquier caso, la presencialidad en este tipo de asignaturas resulta fundamental.

El resto de este artículo se organiza en la forma que se indica a continuación: la siguiente sección trata de justificar el uso de microvídeos en el aprendizaje de los conceptos básicos de control automático, en la sección 3 se describe el proceso de preparación, diseño y publicación de los contenidos desarrollados, en la sección 4 se presentan y discuten algunos resultados preliminares, por último, en la sección 5 , se presentan las conclusiones finales de este trabajo y se comentan ciertos aspectos en los que se pretende seguir trabajando.

\section{JUSTIFICACIÓN}

A raíz de los primeros resultados observados tras la implantación de los planes de estudio de grado, el profesorado del Dpto. de ISA de la UMA se ha venido coordinando para homogeneizar los conceptos teórico-prácticos impartidos y perfeccionar el proceso de enseñanza-aprendizaje con el fin de mejorar los resultados académicos. A consecuencia de ello, se introdujeron bastantes cambios metodológicos en la docencia de estas asignaturas: tutorías grupales, realización de trabajos académicos a lo largo del curso, incentivación de la participación en clase del alumnado, creación/gestión de foros, redacción y publicación de FAQs, etc. Sin embargo, pese a los cambios metodológicos introducidos, no se produjo la mejoría en los resultados académicos que cabría esperar.

A la vista de estos resultados, se llegó a la conclusión de que existen problemas de fondo, no relacionados directamente con las estrategias de enseñanzaaprendizaje utilizadas, que impiden que el alumno asimile los fundamentos necesarios para construir adecuadamente sus conocimientos. En el curso académico 2016/2017 se trató de analizar en detalle esta problemática, identificándose las siguientes posibles causas para esos problemas:

A. la dificultad intrínseca de los conceptos que se abordan,
B. el insuficiente tiempo disponible para plantear y solucionar problemas en clase,

C. la dificultad del alumnado para entender y asimilar los conceptos al ritmo en que se imparten,

D. la imposibilidad de abordar en las sesiones prácticas todos los conceptos explicados en las sesiones de teoría,

E. la dificultad del alumnado para distinguir, pese al esfuerzo de los profesores, lo que es relevante de lo que no lo es, y por último,

F. se han percibido cambios en el modo en que el alumnado espera que se le comunique la información.

A la vista de los problemas identificados y ante la imposibilidad de hacer frente a la totalidad de ellos, se planteó la posibilidad de abordar la solución de algunos de ellos con el fin de minimizar su influencia en el rendimiento del estudiante. Los autores propusieron la creación de nuevos contenidos didácticos que permitieran atacar los problemas A, B, $\mathrm{C}$ y $\mathrm{E}$ y hacerlo con herramientas que resultaran atractivas para el estudiante (problema F). Una de esas herramientas es, según la opinión de éstos, el microvídeo.

El empleo de microvídeos puede reforzar el aprendizaje presencial del alumnado y mejorar su rendimiento académico [11]. Esta creencia se justifica, en primer lugar, en la consideración de que esta herramienta puede hacer más atractivo el aprendizaje $\mathrm{y}$, por tanto, propiciar el interés del alumnado por el mismo; en segundo lugar, el alumnado puede visionarlo en ausencia del profesor y esto habilita, por su flexibilidad y posibilidad de repetición de actuaciones (se puede parar y revisar los vídeos tantas veces como se quiera) que cada alumno pueda tener un mayor control de su proceso de aprendizaje al poder elegir su propio ritmo de seguimiento y asimilación de contenidos en función de su capacidad y circunstancias personales.

\section{DISEÑO, CREACIÓN Y DISTRIBUCIÓN DE LOS MICROVÍDEOS}

En internet hay gran cantidad de vídeos que abordan aspectos relacionados con el control automático [4]. Sin embargo, lo hacen de forma aislada, sin organización alguna, sin un enfoque práctico y sin ejemplos resueltos con software específico como la Control System Toolbox de MATLAB [8] (herramienta de referencia en el área de conocimiento considerada), y lo que es más grave, en nuestra opinión, sin el suficiente rigor científico-técnico.

Los alumnos también pueden encontrar clases grabadas impartidas por profesores [6][7], 
OpenCourseWares [5] y MOOCs [1][9], que, pese a que sí gozan del correspondiente rigor científicotécnico, no resultan suficientemente atractivos para el alumno cuando se utilizan como material de apoyo o refuerzo ya que, por lo general, suelen ser excesivamente largos (entre 30 y $60 \mathrm{~min}$.), lo cual obliga a filtrar contenidos, o resultan poco eficaces sin la correspondiente explicación del profesor.

En este trabajo se ha puesto atención tanto en la presentación (el formato, el diseño de contenidos, la herramienta de creación) y la organización como en el fondo, abordando gran parte de los conceptos que se imparten en las asignaturas indicadas y aportando, cuando procedía, ejemplos teórico-prácticos resueltos con herramientas informáticas adecuadas, y todo ello se ha ejecutado con un estilo homogéneo y bajo la apariencia de un canal temático.

Estas cuestiones suponen la principal contribución de este trabajo cuando se comparan sus resultados con el material relacionado que es posible encontrar en Internet.

\subsection{PREPARACIÓN Y CREACIÓN DE LOS CONTENIDOS}

Esta fase supuso el núcleo central del trabajo realizado y consistió, fundamentalmente, en identificar los conceptos básicos teóricos y prácticos del control automático más problemáticos para el alumnado y elaborar contenidos relacionados con éstos. Las tareas llevadas a cabo fueron las siguientes:

1. Identificación de los contenidos a tratar. Tomando como punto de partida la experiencia de los autores, se seleccionaron los conceptos básicos relacionados con el control automático de sistemas en tiempo continuo que entrañan una mayor dificultad para el alumnado. A continuación, se detallan los bloques conceptuales considerados:

a. Descripción externa e interna de sistemas.

b. Modelado de sistemas dinámicos.

c. Respuesta temporal de sistemas lineales e invariantes en el tiempo (LTI).

d. Respuesta en frecuencia de sistemas LTI.

e. El lugar de las raíces de un sistema realimentado.

f. Introducción al diseño de sistemas de control.

2. Desarrollo de los guiones. Los contenidos elaborados cubrían, además de los aspectos básicos del control automático identificados en el punto 1, ejemplos teórico-prácticos y resolución de problemas en ordenador utilizando MATLAB. Asimismo, se procuró que la duración de los microvídeos no excediera los 5 minutos (una elevada duración puede provocar que el alumno abandone), lo cual obligó a fragmentar cuidadosamente los contenidos e idear una organización eficaz de los mismos.

3. Selección de la herramienta de edición. Para la edición de contenidos se utilizó VideoScribe [14], una herramienta de edición que permite la creación de contenidos vistosos, modernos y amenos. A la hora de elegir la herramienta, se tuvo en cuenta su facilidad de uso, el coste de la licencia y los recursos disponibles [13].

\subsection{PUBLICACIÓN Y DIFUSIÓN DE LOS CONTENIDOS}

Desarrollar contenidos docentes de calidad es importante, pero no sirve de nada si no se hacen llegar al alumnado de la manera más eficaz posible. Por otra parte, tan importante como su distribución es la organización de los mismos, dado que, una organización no coherente, podría propiciar la confusión del alumno a la hora de emplearlos. En relación con esta cuestión se tomaron las siguientes decisiones:

1. Plataforma de distribución. Para la distribución de los microvídeos se optó por YouTube [15]. Esta plataforma cuenta con herramientas sociales, moderación de comentarios, estadísticas, etc. y, además, es gratuita. Por otro lado, existen aplicaciones nativas para el acceso a esta plataforma desde todo tipo de dispositivos: smartphones, tablets, smart tvs, etc., lo que facilita el consumo de los contenidos alojados en la misma en cualquier lugar $\mathrm{u}$ ocasión.

2. Organización de los contenidos. Los vídeos creados se han agrupado según su temática mediante listas de reproducción. Adicionalmente, se han creado listas de reproducción alternativas (itinerarios) para dirigir a los alumnos de una determinada titulación a microvídeos específicos en los que se abordan conceptos no impartidos en otras titulaciones. De esta forma, por ejemplo, las listas correspondientes al bloque temático "Descripción externa e interna de sistemas" no son exactamente las mismas para los alumnos de una titulación que para los alumnos de otra.

También se han utilizado enlaces insertados en los microvídeos para mostrar alternativas al alumnado. Así, por ejemplo, el alumno 
puede estar reproduciendo un vídeo sobre la obtención de la función de transferencia de un circuito RLC a través de sus ecuaciones diferenciales y durante su reproducción ser mostrados enlaces a microvídeos que, o bien resuelven el mismo problema, pero empleando impedancias complejas, o bien analizan la respuesta temporal de un sistema de segundo orden.

Esta organización de los contenidos aporta numerosas ventajas: el alumnado dispone de una lista que incluye todos los vídeos relacionados con cierta temática, pero, además, puede organizar su aprendizaje según sus necesidades, ya que durante su visualización se le ofrece la posibilidad de reproducir microvídeos que pueden estar relacionados con el vídeo en curso y no estar incluidos en la misma lista de reproducción.

3. Difusión de los microvídeos. Para dar a conocer a los estudiantes los contenidos elaborados, se optó por publicar, en los espacios virtuales de cada asignatura, avisos y enlaces, tanto para los microvídeos como para las listas de reproducción. Esto aporta una ventaja doble, pues permite publicitar los microvídeos de forma ordenada y de acuerdo con la evolución de las clases presenciales y, simultáneamente, realizar un seguimiento del empleo de los mismos por parte de los estudiantes a partir de los informes correspondientes proporcionados por el propio campus virtual de la UMA (que está basado en Moodle [10]).

\section{RESULTADOS Y DISCUSIÓN}

El desarrollo y publicación de los contenidos elaborados se inició a principios de 2017 (www.youtube.com/automaticabasica) y continúa en la actualidad. Los primeros contenidos fueron puestos a disposición de los alumnos de tres asignaturas obligatorias de diferentes titulaciones de grado, una de ellas impartida en el primer semestre del curso indicado y las otras dos, en el segundo. Al margen de lo indicado, este proceso se llevó a cabo de forma progresiva, por lo que no fue hasta finales de julio del 2017 cuando se pudo contar con un número representativo de microvídeos, momento en que las clases del curso indicado ya habían finalizado. Como consecuencia de esto, fue imposible cuantificar adecuadamente impacto de los mismos en los 350 alumnos matriculados en estas asignaturas.

Sin embargo, al final del primer semestre de dicho curso, un número significativo de alumnos de la primera asignatura en la que se ofertó una parte de los contenidos elaborados (la disponible entonces) participó en la cumplimentación de unas encuestas de satisfacción (ver Figura 1) que fueron concebidas con la intención de conocer su opinión y tener la oportunidad de detectar y subsanar las deficiencias que pudieran haber sido observadas por éstos en el empleo de los microvídeos. En ese momento estos materiales estaban accesibles en un servidor de la UMA, no en YouTube, donde empezaron a publicarse los vídeos una vez conocidas las valoraciones $y$ sugerencias recogidas en estas encuestas [2].

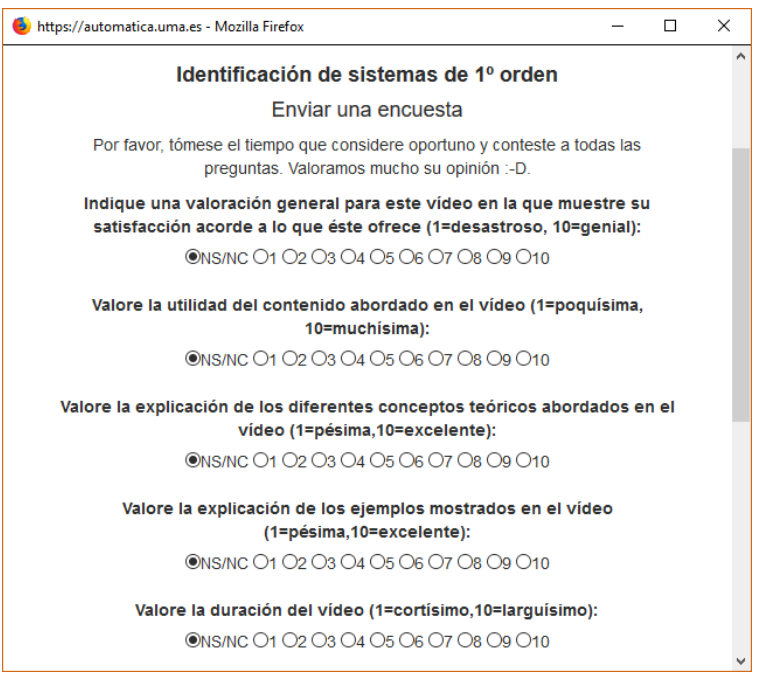

Figura 1: Encuesta voluntaria asociada al microvídeo "Identificación de sistemas de primer orden".

Al inicio del curso académico 2017/2018 se realizó un importante esfuerzo de coordinación en el que participaron todos los profesores del Dpto. de ISA que imparten asignaturas en las que se tratan conceptos básicos del control automático, el cual finalizó con la publicación de los contenidos en 14 asignaturas. Para facilitar esta tarea se crearon recursos compatibles con Moodle que fueron importados en cada asignatura y que contenían enlaces a una aplicación WEB desarrollada exprofeso para este proyecto. Esta aplicación es la encargada de servir los vídeos de YouTube (utilizando la API proporcionada por la plataforma) y recolectar estadísticas de acceso, duración de cada reproducción, pausas y repeticiones realizadas por los alumnos, sugerencias, avisos de erratas, etc., así como una breve encuesta sobre diferentes aspectos del vídeo (duración, estilo, timing -velocidad de las explicaciones-, etc.) (ver Figura 2).

La información recopilada por la plataforma ha sido analizada con objeto de extraer datos significativos a diferentes niveles: mejora de los contenidos, planificación de la producción de nuevos contenidos, etc. Así, por ejemplo, con el análisis de esta información se ha podido: 


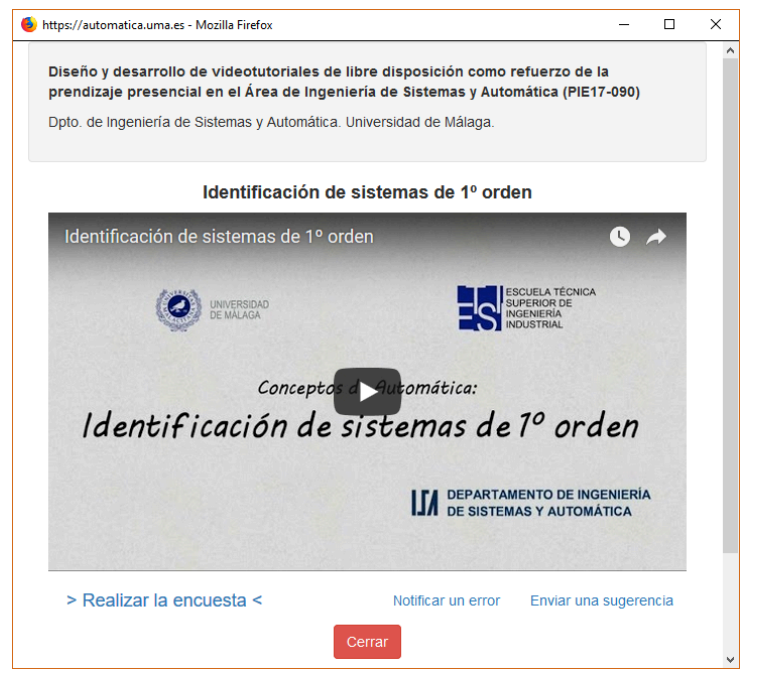

Figura 2: Vídeo mostrado a través del servidor Web encargado de recolectar los datos de uso por los alumnos.

- Corregir posibles errores a partir del feedback de los alumnos.

- Ajustar la velocidad a la que se muestran las explicaciones mediante el análisis de las pausas o retornos.

- Detectar vídeos "problemáticos" (poco atractivos, mal diseñados, con conceptos mal explicados, etc.) mediante el análisis de la tasa de abandono (relación entre el número de reproducciones no finalizadas y el número total de ellas).

- Identificar la tipología (ejercicio, ejemplo, demo con MATLAB, explicación de un concepto teórico, etc.) y el bloque conceptual de los vídeos más demandados.

Además de una mejora de los materiales disponibles en las asignaturas consideradas, el objetivo principal que se han marcado los autores con el desarrollo de estos microvídeos ha sido la mejora de los resultados académicos de los alumnos que han hecho uso de ellos a lo largo del curso.

Extraer conclusiones fiables sobre el rendimiento académico entraña numerosas dificultades ya que, como es obvio, el rendimiento académico de un alumno depende de múltiples factores y no sólo de los recursos que éste tiene a su disposición. Además, cualquier análisis en este sentido requiere de una gran población y debe abarcar un periodo de tiempo superior al considerado aquí. Por último, a lo anteriormente referido, habría que añadir, también, la dificultad técnica para cruzar las calificaciones obtenidas por los alumnos en las diferentes pruebas evaluativas realizadas durante el curso con la información recabada en la aplicación.
No obstante, con este objetivo en mente, se han analizado los resultados académicos obtenidos por los alumnos de la asignatura Fundamentos de Control Automático (sólo ha sido posible realizar, por el momento, dicho análisis en esta asignatura donde todas las pruebas de evaluación están informatizadas), perteneciente al Grado en Ingeniería de la Energía impartido en la Escuela de Ingenierías Industriales de la Universidad de Málaga, y se han cruzado con los datos registrados por la plataforma Web. A continuación se detallan los resultados obtenidos más relevantes:

a. De las 4990 reproducciones (sólo se contabilizan las realizadas a través del servidor Web) realizadas durante el presente curso, 1426 corresponden a la referida asignatura. De los 60 alumnos matriculados, 43 reprodujeron al menos 1 vídeo $(71.6 \%)$ y sólo 10 reprodujeron 25 vídeos o más $(16.6 \%)$, en una o en diversas ocasiones.

b. La nota media obtenida en el examen correspondiente a la primera convocatoria ordinaria por los alumnos que han reproducido al menos una vez 25 vídeos o más ha sido de $6.81( \pm 1.37)$, mientras que la nota media obtenida por los alumnos que han reproducido al menos una vez 15 vídeos o menos es de $6.13( \pm 0.93)$. Estos resultados resultan esperanzadores, pero para que fueran concluyentes se requeriría un análisis más exhaustivo y con una mayor muestra.

c. Los 3 vídeos más reproducidos, con cerca de 75 reproducciones cada uno, pertenecen a las listas de reproducción "Respuesta en frecuencia de sistemas LTI" y "Lugar de las raíces", que se corresponden, a su vez, con los bloques conceptuales con mayor carga lectiva de la asignatura y que entrañan más dificultades para los alumnos.

d. Según las opiniones recabadas de forma voluntaria: el $95.9 \%$ de los alumnos recomendarían los microvídeos a sus compañeros, el $86.4 \%$ los valoran positivamente, el $76.2 \%$ consideran que la duración es la apropiada y el $86.6 \%$ valoran positivamente el contenido abordado en ellos.

Los autores consideran que los resultados obtenidos, pese a ser escuetos, abarcar un solo curso académico y disponer, por el momento, del análisis de datos más concretos de una sola asignatura, son prometedores e ilustran la utilidad de estos contenidos como material de apoyo para el alumno. Asimismo, los autores creen que éstos podrían interesar a otros docentes que impartan asignaturas parecidas. 


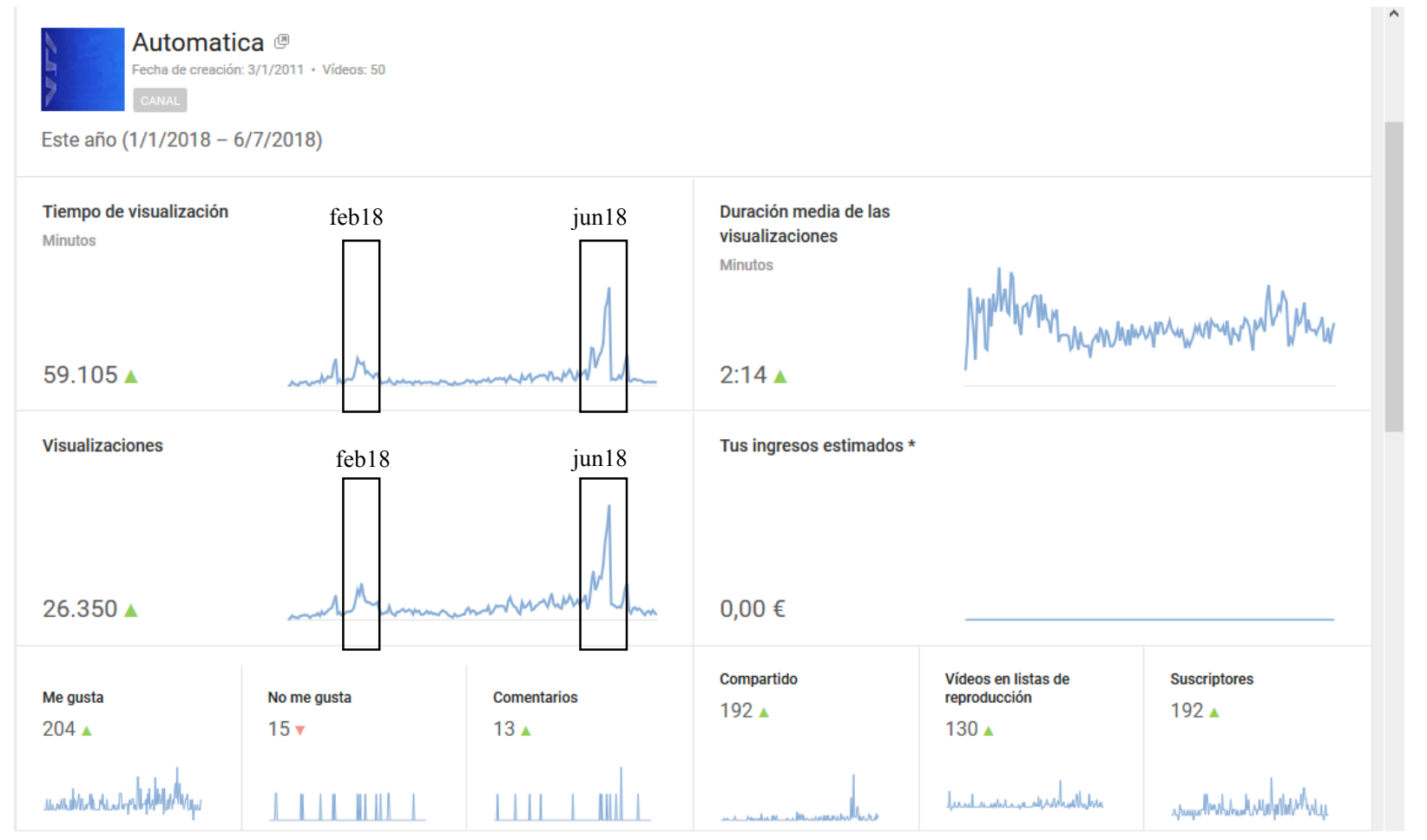

Figura 3: Tiempo de visualización, duración media de las reproducciones, número de reproducciones y otros datos del canal de YouTube (www.youtube.com/automaticabasica) desde el 1 de enero al 6 de julio de 2018.

La Figura 3 recoge una serie de estadísticas proporcionadas por YouTube que ofrecen una visión bastante esclarecedora de la evolución de diversos parámetros del canal de esta plataforma en el que han sido publicados los microvídeos elaborados a lo largo de esta experiencia de innovación educativa. El periodo de tiempo considerado en dichas estadísticas va desde el comienzo del mes de enero hasta el día 6 de julio de 2018. En dichas gráficas se puede observar que en este intervalo temporal se producen unos incrementos muy importantes, con respecto al periodo anterior, del tiempo de visualización, la duración media de las reproducciones y las visualizaciones del canal. Asimismo, resulta interesante ver cómo el tiempo de visualización y el número de visualizaciones se disparan en las fechas próximas a los exámenes de la primera convocatoria ordinaria (febrero o junio) de las asignaturas involucradas en este trabajo.

En la actualidad el canal cuenta con 39 microvídeos, 9 listas de reproducción (bloques conceptuales e itinerarios), un total de 564 suscriptores, 55.325 reproducciones, 78 días y 6 horas de tiempo acumulado de visualización y es partner de YouTube (insignia que proporciona YouTube a los canales con muchos suscriptores y un mínimo de 2000 visitas diarias). Estos microvídeos se utilizan en un total de 14 asignaturas impartidas por el Dpto. de ISA de la
UMA como recurso de apoyo a la docencia y pueden ser "consumidos" desde multitud de plataformas.

\section{CONCLUSIONES Y FUTUROS TRABAJOS}

En este artículo se recoge una experiencia de innovación educativa, realizada durante el curso 2017/2018 en el Dpto. de ISA de la UMA, consistente en el empleo de microvídeos, accesibles en un canal de YouTube, como herramienta de apoyo para el aprendizaje de los conceptos básicos del control automático por parte de los alumnos de 14 asignaturas de diferentes titulaciones. Dicho aprendizaje suele entrañar una gran dificultad al alumnado, por lo que un aspecto clave en esta experiencia ha sido la búsqueda de estrategias para que los materiales elaborados resultaran amenos y atractivos, a la vez que didácticos. Al margen de esto, el empleo de un canal de YouTube para su publicación se justifica en que este espacio es un medio de comunicación ampliamente utilizado por el alumnado y que está disponible para multitud de plataformas (teléfonos móviles, PCs, tabletas, videoconsolas y TVs).

Por otra parte, el análisis de las encuestas de satisfacción del alumnado relativas a los microvídeos disponibles que han sido realizadas a lo largo del curso, así como el estudio de los datos registrados en 
la Web diseñada para el proyecto ha arrojado resultados muy prometedores: unas mejores calificaciones medias obtenidas por el alumnado que ha hecho uso de los materiales en comparación con el resto, así como un incremento en el número de reproducciones y de valoraciones positivas de los contenidos. También se ha observado un aumento sustancial en el tiempo de visualización, el número de "me gusta" y el número suscriptores del canal de YouTube, lo cual pone de relieve el interés que han puesto los estudiantes en los materiales aquí descritos que les han sido proporcionados.

Los autores consideran que los resultados recogidos en este trabajo son ilustrativos, pero no concluyentes. Es conveniente profundizar en el análisis del impacto del empleo de los videos en el rendimiento académico del alumnado, lo cual obligaría a relacionar el uso de éstos con los resultados de las pruebas de evaluación de un mayor número de alumnos y durante un mayor periodo de tiempo. Por último, sería interesante crear nuevos vídeos con otros contenidos, por ejemplo, el control automático en tiempo discreto o pruebas experimentales realizadas en laboratorio que ayuden al alumnado a entender conceptos teóricos o prácticos.

\section{Agradecimientos}

Los autores desean dar las gracias al profesorado del Dpto. de Ingeniería de Sistemas y Automática de la Universidad de Málaga que ha colaborado desinteresadamente en el desarrollo de este Proyecto de Innovación Educativa.

Este trabajo ha sido financiado a través del Plan de Innovación Educativa de la Universidad de Málaga (convocatoria 2017/2019) bajo la referencia PIE17090 .

\section{English summary}

\section{THE USE OF MICROVIDEOS AS REINFORCEMENT OF FACE-TO- FACE LEARNING IN TEACHING OF AUTOMATIC CONTROL BASIS}

\author{
Abstract \\ This work describes an educational innovation \\ experience, carried out during the course 2017/2018 \\ in the Dept. of Systems Engineering and Automation \\ of the University of Malaga. This experience has \\ consisted in the use of microvideos as a support tool \\ for learning the basic concepts of continuous time \\ systems automatic control by students of 14 subjects \\ of different graduate studies.
}

In general, students find great difficulties in such learning, so a key aspect in this experience has been the search for strategies so that the materials developed would be entertaining and attractive, as well as didactic.

In order to facilitate, promote and analyze the use of the developed contents, the advantages of using a platform such as YouTube have been exploited. Currently, the www.youtube.com/automaticabasica channel, which has included the produced material, has 39 microvideos, 9 playlists (conceptual blocks), a total of 564 subscribers, 55,325 reproductions and approximately 78 days of accumulated play time. The obtained results of this experience, although preliminary, show a positive impact on the academic performance of the students who have used them.

Keywords: automatic control, autonomous learning, microvideo, YouTube, MATLAB, educational innovation.

\section{Referencias}

[1] Albertos, P. (2018), Dynamics and Control. Interactive course about the basic concepts of Systems, Control and their impact in all the human activities. MOOC Universitat Politècnica de Valencia.

[2] Arévalo, V.; Vicente del Rey, J.M. y Rodríguez, H. (2017), "Creación de videotutoriales como refuerzo del aprendizaje presencial en el Área de Ingeniería de Sistemas y Automática", $V$ Jornadas Internacionales sobre Innovación Docente en las Titulaciones Técnicas (INDOTEC'17), Granada (Spain).

[3] Calle, D. Canal de YouTube UNICOOS (Matemáticas, Química, Física, etc.). https://www.youtube.com/user/davidepv (consultada el 03/06/2018).

[4] Colmenares, W. Canal de YouTube (lugar de las raíces y modelado de sistemas físicos). https://www.youtube.com/user/ktulu1988 (consultada el 05/06/2018).

[5] Hall, S. (2012), Principles of Automatic Control. Massachusetts Institute of Technology: MIT OpenCourseWare, https://ocw.mit.edu.

[6] Douglas, B. Canal de YouTube (control system lectures). https://www.youtube.com/channel/UCq0imsn84 ShAe9PBOFnoIrg. (consultada el 05/07/2018) 
[7] Itfigueres. Canal de YouTube (lugar de las raíces y trazado asintótico de diagramas de Bode). https://www.youtube.com/channel/UCL--

GdIszTsqTPJDdC8cxYQ. (consultada el 01/06/2018).

[8] MATLAB. Control System Toolbox web page. https://es.mathworks.com/products/control.html (consultada el 01/07/2018).

[9] Maya-Ortiz, P.R.; Arteaga-Pérez M.A.; González-Olvera, and M.Á. (2018), Control automático: La tecnología invisible. MOOC.es.

[10] Moodle. Web page. https://moodle.org/. (consultada el 08/07/2018).

[11]Rodenas, M. (2012), "La utilización de los vídeos tutoriales en educación. Ventajas e inconvenientes. Software gratuito en el mercado". Revista Digital Sociedad de la Información, 33.

[12] Sugimoto, C.R.; Thelwall, M.; Larivière, V.; Tsou, A.; Mongeon, P. and Macaluso, B. (2013). "Scientists Popularizing Science: Characteristics and Impact of TED Talk Presenters". PLOS ONE, 8(4).

[13] VideoScribe tutorials and training. YouTube playlist.

https://www.youtube.com/playlist?list=PL941uN yvzPSHkj3eC FgaVR1F6iaUEGAC.

(consultada el 15/03/2017).

[14] VideoScribe. Web page. http://www.videoscribe.co/ (consultada el 02/07/2018).

[15] YouTube. Web page. https://www.youtube.com/ (consultada el 02/07/2018).

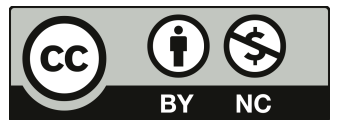

(C) 2018 by the authors. Submitted for possible open access publication under the terms and conditions of the Creative Commons Attribution CC-BY-NC 3.0 license (https://creativecommons.org/licenses/by-nc/3.0). 